\title{
Limit cycles and Hopf bifurcations in a Kolmogorov type system $\dagger$
}

\author{
SIMONA MURATORI $\ddagger$ and SERGIO RINALDI§
}

\begin{abstract}
Keywords: non-linear systems, limit cycle, Hopf bifurcation, Poincaré index, stability, prey-predator models.

The paper is devoted to the study of a class of Kolmogorov type systems which can be used to represent the dynamic behaviour of prey and predators. The model is an extension of the classical prey-predator model since it allows intraspecific competition for the predator's species. The analysis shows that the system can only have Kolmogorov's two modes of behaviour: a globally stable equilibrium or a globally stable limit cycle. Moreover, the transition from one of these two modes to the other is a non-catastrophic Hopf bifurcation which can easily be specified analytically.
\end{abstract}

\section{Introduction}

This paper is concerned with Hopf bifurcations (Marsden and Mc Sracken 1976) and cycles in a second order dynamic system used to model a renewable resource and its consumers. The resource $x$ and the consumers $y$ are also called prey and predators and the corresponding model

$$
\begin{aligned}
& \dot{x}=x f(x, y) \\
& \dot{y}=y g(x, y)
\end{aligned}
$$

is known as the prey-predator model.

If $f(0, y)$ and $g(x, 0)$ are bounded for $x \geqslant 0$ and $y \geqslant 0$, system $(1,2)$ is a positive, dynamic system since $x(0), y(0) \geqslant 0$ implies $x(t), y(t) \geqslant 0$, for all $t>0$. Its properties were first investigated by Lotka and Volterra who showed that an infinity of periodic solutions can exist if $f$ and $g$ are linear. Since then, a variety of particular preypredator models have been proposed and used to describe non-linear oscillations in biology and natural resources management. Among them we can recall the model (from now on, termed the classical model, see $\S 2$ ) in which the growth of the resource is logistic and the consumers are characterized by a finite capacity of handling their prey (the well-known predators' functional response). A large class of these models has either a globally stable equilibrium or a unique unstable and strictly positive equilibrium surrounded by a globally stable limit cycle. These two possible modes of behaviour, conjectured by Kolmogorov in a celebrated paper (Kolmogorov 1936) and later investigated by other authors (Albrecht et al. 1974), seem to be very frequent in prey-predator systems. For this reason it is interesting to point out the exceptions (Muratori and Rinaldi 1989) and establish how a system

Received 29 August 1988.

$\dagger$ Centro Teoria dei Sistemi, CNR, Dipartimento di Elettronica, Politecnico di Milano, 20133 Milano, Italy.

¥ Scientific consultant, Milano, Italy.

$\S$ Professor of System Theory at the Department of Electronics, Politecnico di Milano, and Research Associate at the Centro Teoria dei Sistemi, CNR, Milano, Italy. 
moves from one mode to another when the strategic parameters are varied slightly. This is obviously a bifurcation problem in which the type of the bifurcation is of definite importance.

The paper is organized as follows. In $\S 2$ we recall the main properties of the classical prey-predator model. Then, in $\S 3$ we extend the model by taking an important physical phenomenon into account, namely intraspecific competition among the predators. Finally in $\S 4$ we analyse the stability properties of this new model and prove that there is a non-catastrophic Hopf bifurcation which separates the two possible modes of behaviour. Moreover, we briefly discuss the effects of intraspecific competition and explicitly point out the value of the parameters which give rise to the Hopf bifurcation. This is particularly important, since there are still only a few non-linear systems for which Hopf bifurcations can be computed so as to avoid simulation.

\section{The classical model}

The most commonly used prey-predator model is the following

$$
\begin{aligned}
& \dot{x}=x\left[r\left(1-\frac{x}{K}\right)-\frac{b}{c} \frac{y}{a+x}\right] \\
& \dot{y}=y\left[\frac{b x}{a+x}-d\right]
\end{aligned}
$$

where $r$ and $K$ are the intrinsic growth rate and the carrying capacity of the resource, $b$ and $d(b>d)$ are the maximum birth rate and the constant death rate of the consumers, $a$ is the half saturation constant of the consumers' functional response, and $c$ is a conversion factor. All these parameters are strictly positive.

Model $(3,4)$ has been extensively used in applications and its basic properties are now well known (Hsu, Hubbel and Waltman 1978). Gilpin (1972) was the first to establish, through numerical simulation, that limit cycles can exist for suitable values of the parameters. Shimazu et al. (1972) found the same results and proved through local analysis (see Fig. 1) that the origin is always unstable (saddle point), that the equilibrium $(K, 0)$ (resource at its carrying capacity and absence of

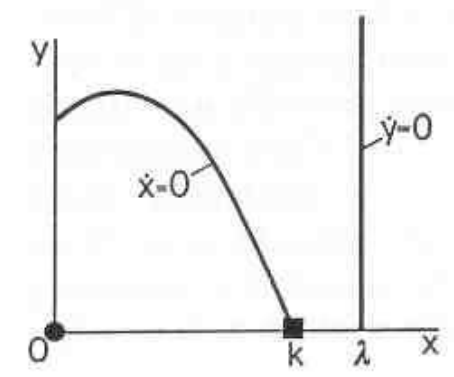

(a)

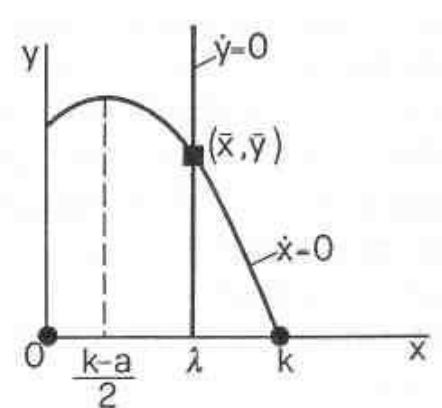

(b)

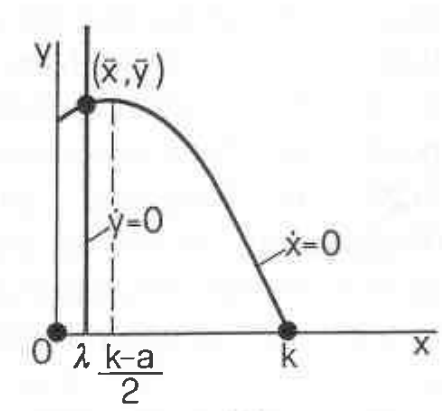

(c)

Figure 1. Non-trivial isoclines $\dot{x}=0, \dot{y}=0$ and stable ( $\square$ ) and unstable (O) equilibria of system $(3,4)$ for different values of the parameters. Case $(a): \lambda=a d /(b-d)>K$; Case (b): $(K-a) / 2<\lambda<K$; Case $(c): 0<\lambda<(K-a) / 2$. (If $K<a$ the vertex of the parabola is in the second quadrant and case (c) is not possible). 
consumers) is stable if and only if

$$
\lambda=\frac{a d}{b-d}>K
$$

and that a positive equilibrium

$$
\bar{x}=\lambda \quad \bar{y}=\frac{c r}{b}\left(1-\frac{\lambda}{K}\right)(a+\lambda)
$$

exists provided $\lambda<K$. Moreover, this equilibrium is stable if (see Fig. 1(b)).

$$
\max \left[\frac{K-a}{2}, 0\right]<\lambda<K
$$

and unstable if (see Fig. 1(c))

$$
0<\lambda<\frac{K-a}{2}
$$

But more can be said on the stability properties of system $(3,4)$. In fact, it can be proved that the equilibrium $(\bar{x}, \bar{y})$ is globally stable in the first quadrant if (7) is satisfied, and that a cycle exists around it when (8) holds. Moreover, this cycle is unique and globally stable in the first quadrant. The proof of the last property (Cheng 1981) is certainly the most difficult part of the analysis and is based on a detailed investigation of the geometric properties of a Liapunov function.

In conclusion, the system can have only Kolmogorov's two modes of behaviour: a globally stable equilibrium (Fig. 1(a) and 1(b)) or a globally stable cycle (Fig. 1(c)). The critical value of the parameter $\lambda$ which separates these two modes is

$$
\lambda^{*}=\frac{K-a}{2}
$$

(notice that this value exists if $K>a$ ). For this value of $\lambda$ there is a Hopf bifurcation (Smith 1982). In fact $f_{x}=0$ and the Jacobian matrix

$$
J=\left|\begin{array}{cc}
x f_{x} & x f_{y} \\
y g_{x} & 0
\end{array}\right|_{\substack{x=\bar{x} \\
y=\bar{y}}}
$$

has a null trace and a positive determinant (notice that $f_{y}<0$ and $g_{x}>0$ ), so that its eigenvalues are purely imaginary.

The analysis is summarised in Fig. 2, which shows the equilibria and the cycles of system $(3,4)$ for all values of parameter $\lambda$. For $\lambda=\lambda^{*}=(K-a) / 2$ and for $\lambda=K$ there are two non-catastrophic bifurcations, the first one being a Hopf bifurcation.

\section{The extended model}

In Eqn. (3) intraspecific competition is taken into account since the prey growth rate per capita in the absence of predators, namely $r(1-x / k)$, is a decreasing function of $x$. This accounts for different phenomena, such as scarcity of food per capita, competition for space, higher chances of epidemics, which, in general, increase mortality at high densities. On the contrary, in Eqn. (4) the death rate $d$ is kept constant. This is a serious drawback of model $(3,4)$ not only with respect to applications, 


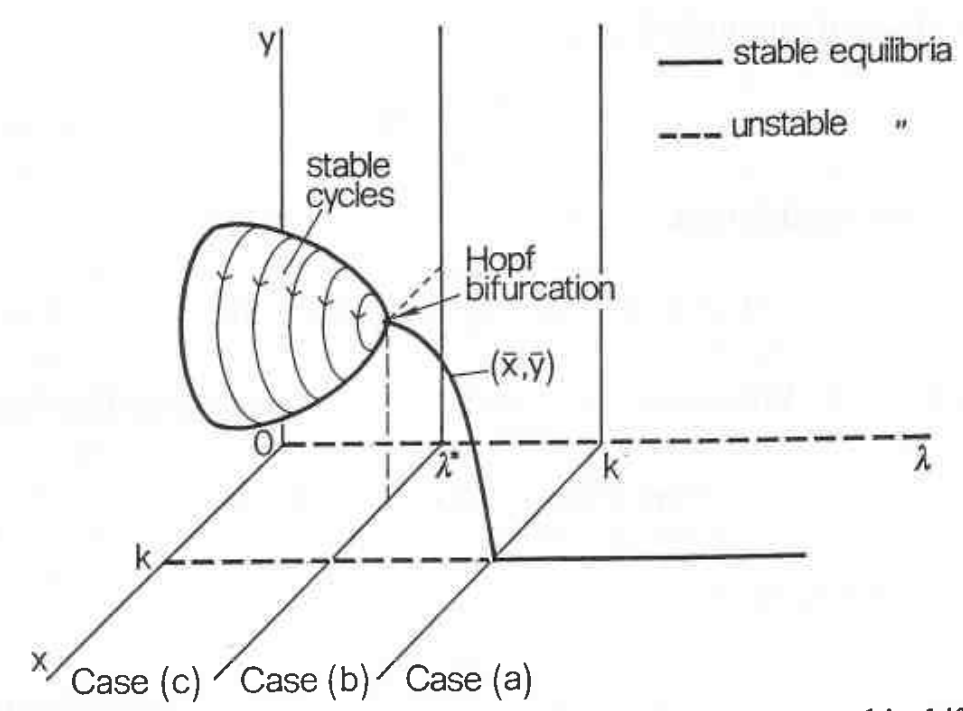

Figure 2. The control-phase portrait of system $(3,4)$. Non catastrophic bifurcations for $\lambda=\lambda^{*}=(K-a) / 2$ and $\lambda=K$. Cases $(a),(b)$ and $(c)$ make reference to Fig. 1 . If $K<a$ the Hopf bifurcation does not exist.

where intraspecific competition may be a relevant factor, but also considering the qualitative theory that prey-predator models pretend to be nowadays. It is therefore of interest to consider the following extended model

$$
\begin{aligned}
& \dot{x}=x\left[r\left(1-\frac{x}{K}\right)-\frac{b}{c} \frac{y}{a+x}\right] \\
& \dot{y}=y\left[\frac{b x}{a+x}-d-e y\right]
\end{aligned}
$$

in which the positive parameter $e$ represents the degree of intraspecific competition among predators. The analysis which follows emphasizes the role of this parameter.

\section{Analysis of the model}

\subsection{Equilibria}

The isocline $\dot{x}=0$ does not depend upon $e$ and is obviously the same as in the classical model (see Fig. 1). On the contrary the non-trivial isocline $\dot{y}=0$, namely $g=0$, depends on $e$ and is given by (recall that $\lambda=a d /(b-d)$ )

$$
y=\frac{1}{e}\left(\frac{b x}{a+x}-d\right)=\frac{a d}{e \lambda} \frac{(x-\lambda)}{(x+a)}
$$

Such isoclines are increasing and concave and rooted at point $(\lambda, 0)$ as shown in Fig. 3. We can therefore consider the following three cases

(a) $\lambda>K$

(b) $\frac{K-a}{2}<\lambda<K$

(c) $0<\lambda<\frac{K-a}{2}$ 


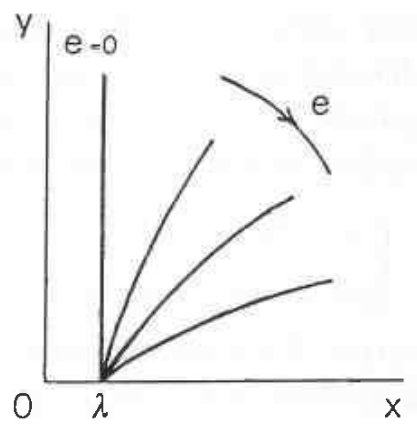

Figure 3. Non-trivial isoclines $\dot{y}=0$ of system $(3,9)$ parametrized with respect to $e$. The isoclines are increasing, concave and rooted at $(\lambda, 0)$.

as was done for the classical model (see Fig. 1 and Eqns. $(5,7,8)$ ).

The first two cases are very simple to analyse. In case $(a)$ there are only trivial equilibria: the origin (unstable) and point $(K, 0)$ (globally stable) (see Figs. $1(a)$ and 3 ). In case $(b)$ (see Figs. $1(b)$ and 3 ), besides the two trivial equilibria which are unstable, there is a stricly positive equilibrium $(\bar{x}(e), \bar{y}(e))$ which is globally stable in the first quadrant (the proof is a trivial extension of the equivalent result known for the classical model). An explicit formula for $(\bar{x}(e), \bar{y}(e))$ cannot be easily obtained since the solution of the system of equations $f=g=0$ can only be found by determining the positive root of a third order polynomial. In any case, Figs. $1(b)$ and 3 show that at the equilibrium the prey increase monotonically with $e$ (from $\lambda=a d /$ $(b-d)$ to their carrying capacity $K)$, while the predators decrease monotonically to zero. This result is rather intuitive and can be explained by saying that when $e$ increases predators spend more energy in competition, thus reducing the stress on the prey.

The analysis of case $(c)$ is more complex: here we make reference to Fig. 4 where the non-trivial isocline $\dot{y}=0$ is drawn for different values of $e$. Again the two trivial equilibria are unstable (saddle points) and there is a strictly positive equilibrium $(\bar{x}(e), \bar{y}(e)$ ). But this equilibrium is either stable (for sufficiently large values of $e$ ( $e>e_{H}$ in Fig. 4, where $e_{H}$ is specified in the following)) or unstable (in the opposite case). In order to prove this, recall that for $e=0$ model $(3,9)$ degenerates into model

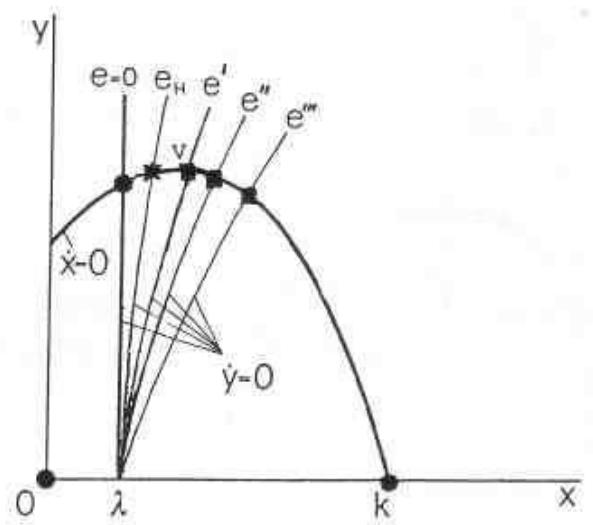

Figure 4. The positive equilibrium $(\bar{x}(e), \bar{y}(e))$ for different values of $e$ when $\lambda<(K-a) / 2$. For $e<e_{H}$ the equilibrium is unstable (O), while for $e>e_{H}$ the equilibrium is stable (a) 
$(3,4)$ so that we know that $(\bar{x}(0), \bar{y}(0))$ is unstable (actually an unstable node or an unstable focus). On the other hand, the nature of the equilibrium $\left(\bar{x}\left(e^{\prime}\right), \bar{y}\left(e^{\prime}\right)\right)$ corresponding to the vertex of the parabola $\dot{x}=0$ (point V in Fig. 4) can be easily established. In fact, at that point $f_{x}=0$ so that the Jacobian matrix

$$
\boldsymbol{J}=\left|\begin{array}{cc}
\bar{x} f_{x} & \bar{x} f_{y} \\
\bar{y} g_{x} & \bar{y} g_{y}
\end{array}\right|=\left|\begin{array}{cc}
0 & \bar{x} f_{y} \\
\bar{y} g_{x} & -e \bar{y}
\end{array}\right|
$$

has a negative trace and a positive determinant since $f_{y}<0$ and $g_{x}>0$. These sign conditions imply that both eigenvalues of $J$ have negative real parts. Thus, the equilibrium $\left(\bar{x}\left(e^{\prime}\right), \bar{y}\left(e^{\prime}\right)\right)$ is either a stable node or a stable focus.

By continuity we could argue that for increasing values of $e$ in the range $\left(0, e^{\prime}\right)$ the nature of the non-trivial equilibrium should change accordingly to one of the two following paths:

(i) (unstable node $\rightarrow$ ) unstable focus $\rightarrow$ stable focus $(\rightarrow$ stable node)

(ii) (unstable focus $\rightarrow$ ) unstable node $\rightarrow$ saddle $\rightarrow$ stable node ( $\rightarrow$ stable focus)

Nevertheless, in the following we prove that the equilibrium $(\bar{x}(e), \bar{y}(e))$ cannot be a saddle so that case (ii) turns out to be unfeasible. Thus, model $(3,9)$ is characterized by a Hopf bifurcation which occurs when the two complex eigenvalues of the Jacobian matrix cross the imaginary axis. At that point the trace of $J$ vanishes while its determinant is positive.

\subsection{Limit cycle}

We now show that the positive equilibrium $(\bar{x}(e), \bar{y}(e))$ is surrounded by a limit cycle whenever it is unstable (the proof is essentially a direct consequence of the well-known Bendixon-Poincaré argument). For this consider the closed line OKPQ (see Fig. 5) comprised of the horizontal straight line PQ and the segments of trajectories $\mathrm{QO}, \mathrm{OK}$, and $\mathrm{KP}$, the last one being the separatrix associated with the positive eigenvalue of the saddle point $(K, 0)$. Trajectories starting inside the region

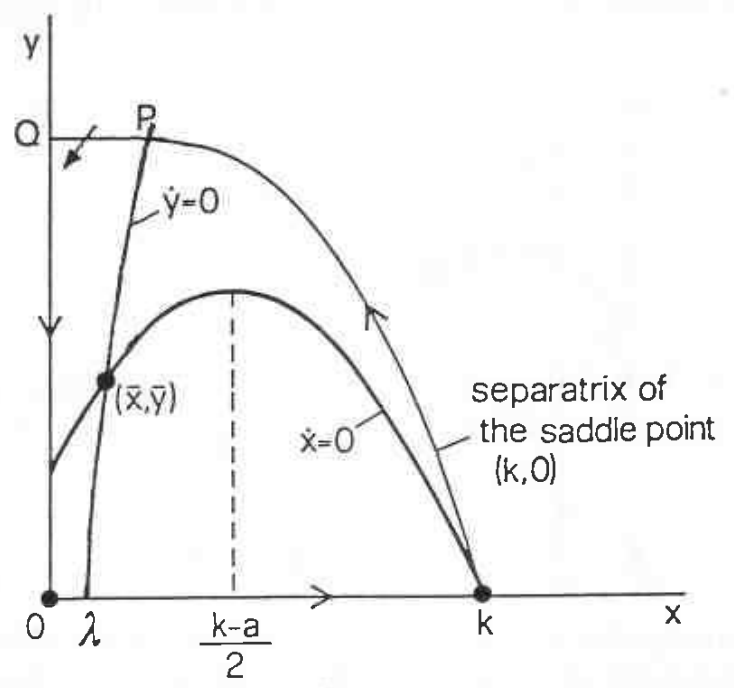

Figure 5. Non-trivial isoclines $\dot{x}=0$ and $\dot{y}=0$ and unstable ( $)$ equilibria of system $(3,9)$. 
cannot cross or tend toward its boundary (notice that $\dot{y} \leqslant 0$ on segment $P Q$ ) and cannot tend to $(\bar{x}(e), \bar{y}(e))$ because, by assumption, such an equilibrium is unstable. Therefore, the trajectories tend toward a limit cycle surrounding the positive equilibrium (the formal proof of global stability and uniqueness of this cycle should be done by extending the proof known for the classical model (Cheng 1981), but this does not seem to be an easy task).

Notice that the existence of a limit cycle around $(\bar{x}(e), \bar{y}(e))$ proves that such an equilibrium cannot be a saddle, as mentioned at the end of the subsection on equilibria. In fact the well-known theorem on Poincare indices says that if there is only one equilibrium inside a closed orbit it must be a node or a focus.

\subsection{Hopf bifurcation}

The stability analysis of the equilibria and the existence of a limit cycle allow us to conclude that system $(3,9)$ has a Hopf bifurcation with respect to the parameter characterizing intraspecific competition if and only if inequality (8) holds. Moreover, the bifurcation is non-catastrophic since the limit cycle exists when the nontrivial equilibrium is unstable. Therefore, one can represent the behaviour of system $(3,9)$ with the simple control-phase portrait shown in Fig. 6 . When $e=0$ we have the limit cycle of the classical model. For increasing values of $e$ this cycle shrinks smoothly until it degenerates to the point $\left(\bar{x}_{H}, \bar{y}_{H}\right)$ for $e=e_{H}$ (the triplet $\left(\bar{x}_{H}, \bar{y}_{H}, e_{H}\right)$ characterizes the Hopf bifurcation). For higher values of $e$ we have only one globally stable equilibrium which asymptotically tends for $e$ going to infinity towards the unstable trivial equilibrium $(K, 0)$. This control-phase portrait clearly indicates that intraspecific competition acts as a damping factor in the overall behaviour of the system.

Now that we have recognized that the key feature of system $(3,9)$ is the existence of a Hopf bifurcation, it is worth showing how the value $e_{H}$ of the parameter can be explicitly determined. For this we must find the positive solution $\left(\bar{x}_{H}, \bar{y}_{H}, e_{H}\right)$ of the

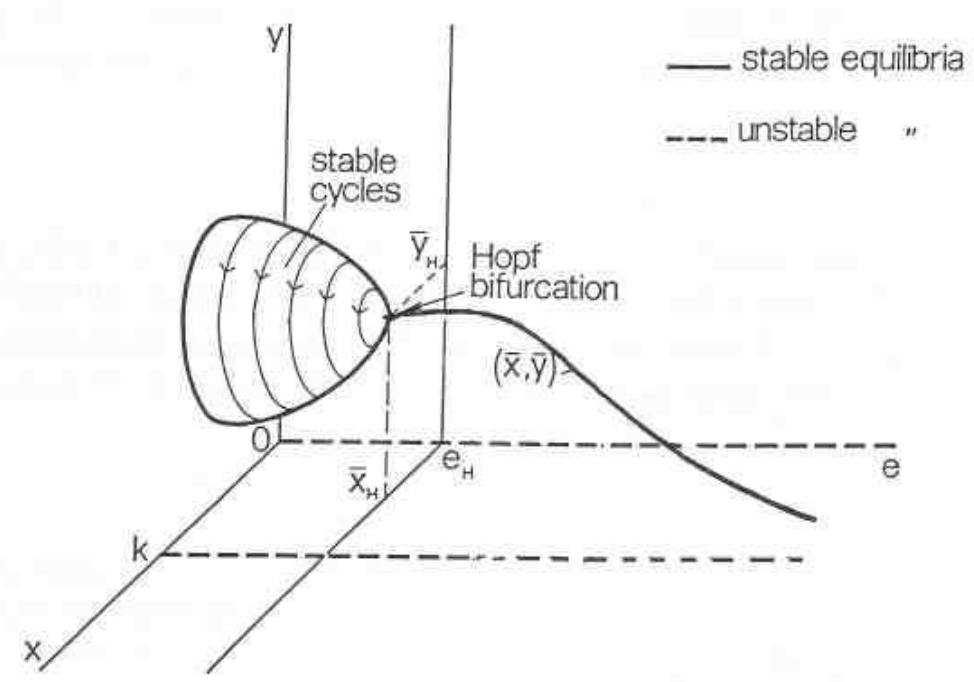

Figure 6. The control-phase portrait of system $(3,9)$ when $\lambda<(K-a) / 2$. The triplet $\left(\bar{x}_{H}\right.$, $\left.\bar{y}_{H}, e_{H}\right)$ defines the Hopf bifurcation. 
following three equations

$$
\begin{array}{r}
f(x, y)=0 \\
g(x, y, e)=0 \\
x f_{x}(x, y)+y g_{y}(x, y, e)=0
\end{array}
$$

this last one stating that the trace of the Jacobian matrix must vanish at the bifurcation. By eliminating $y$ from (11) and (12) and solving with respect to $e$ one obtains (easy to check)

$$
e_{H}=\frac{K a d^{2}}{r c \lambda^{2}} \frac{\left(\bar{x}_{H}-\lambda\right)(a+\lambda)}{\left(K-\bar{x}_{H}\right)\left(a+\bar{x}_{H}\right)^{2}}
$$

which can be used to compute $e_{H}$ once $\bar{x}_{H}$ is known. On the other hand, one can first eliminate $e$ by subtracting (12) from (13) and then take (11) into account to eliminate $y$. This gives rise to the following second order polynomial

$$
p(x)=2 r \lambda x^{2}+(K a d+a r \lambda-K r \lambda) x-\operatorname{Kad} \lambda
$$

which has only one positive root, namely $\bar{x}_{H}$. Thus, given the numerical values of the parameters of the classical model one can determine $\bar{x}_{H}$ by finding the positive root of the polynomial $p(x)$, and then use (14) to determine $e_{H}$ and (10) to determine $\bar{y}_{H}$.

\section{Concluding remarks}

In this paper we have analysed the properties of a second order positive dynamic system which models a renewable resource and its consumers in the case of intraspecific competition. We have shown how this system differs from the classical preypredator model in which intraspecific competition among the consumers is not allowed. In particular it has been proved that there are only two modes of behaviour, as argued long ago by Kolmogorov. These modes are a globally stable equilibrium and a globally stable limit cycle and the transition between the two modes is a non-catastrophic Hopf bifurcation. Surprisingly, the values of the parameters giving rise to such bifurcation can be easily computed avoiding simulation.

\section{ACKNOWLEDGMENTS}

This research was supported by Centro Teoria dei Sistemi, CNR, and by the Italian Ministry of Public Education. The work was done at the Division of Engineering Cybernetics, the Norwegian Institute of Technology, Trondheim, Norway, where the second author spent part of the summer 1988 as a NATO Senior Fellow.

\section{REFERENCES}

Albrecht, F., Gatzke, H., Haddad, A., and Wax, N. (1974). The dynamics of two interacting populations. Journal of Mathematical Analysis and Applications, 46, 658-670.

CHenG, K. S. (1981). Uniqueness of a limit cycle for a predator-prey system. SIAM Journal on Mathematical Analysis, 12, 541-548.

GiLPIN, M. E. (1972). Enriched predator-prey systems: theoretical stability. Science, 177, 902904. 
Hsu, S. B., Hubbel, S. P., and Waltman, P. (1978). Competing predators. SI AM Journal on Applied Mathematics, 35, 617-625.

Kolmogorov, A. M. (1936). Sulla teoria di Volterra della lotta per l'esistenza. Giornale dell' Istituto Italiano degli Attuari, 7, 74-80.

Marsden, J. E., and MCCraCKEN, M. (1976). The Hopf Bifurcation and its Applications (Springer-Verlag, New York).

MuratORI, S., and RinALDI, S. (1989). A dynamical system with Hopf bifurcations and catastrophes. Applied Mathematics and Computation, 29, 1-15.

Shimazu, Y., Sugrama, K., Kojima, T., and Tomida, E. (1972). Some problems in ecology oriented environmentology, Journal of Earth Science, 20, 31-89.

SмITH, H. L. (1982). The interaction of steady state and Hopf bifurcations in a two-predatorone prey competiton model. SIAM Journal on Applied Mathematics, 42, 27-43. 